\title{
Cytotoxic T cells still recognize SARS-CoV-2 Omicron variant even when antibodies cannot
}

\section{Hatem Tallima, Rashika El Ridi}

Cytotoxic T cells still recognize SARS-CoV-2 Omicron variant even when antibodies cannot $^{1}$, because the numerous mutations in the spike glycoprotein (S) are mostly located in subunit 1 (S1), which is shed upon invasion of structural cells, and thus is poorly presented on the surface membrane in association with HLA class I molecules. Accordingly, mutated or not, S1 peptides are not a predominant target for activated or memory cytotoxic $\mathrm{T}$ cells. Vaccine- or previous infection-induced antibodies specific to the $\mathrm{S}$ receptor binding domain may not access linear or conformational determinants the virus has mutated. Conversely, cytotoxic T cells stimulation and killer function are largely under the host control. Upon entry into the cytoplasm of structural cells, virus peptides are generated following virus proteins degradation in the proteasome whose activity differs between cell types and between individuals. Only peptides of the correct size may bind to HLA class I molecules for presentation on the surface membrane, but must follow the restrictions of the HLA molecules, which immensely vary among individuals. Finally, the repertoire of peptides' T cell recognition is genetically determined ${ }^{2}$. This submission to the dictates of the host immune system renders cytotoxic T cells killing rather insensitive to virus mutations, and totally unscathed if the mutations reside predominantly in S1 for the reason mentioned above.

Vaccine- and infection-generated cytotoxic T cells will efficiently kill all variantinfected cells, but that is not recommended in the case of SARS-Cov-2, which is able to invade most cell types, including nervous and endocrine systems key cells, no one 
can replace or compensate $e^{3}$. Additionally, killing infected cells at unpredictable moments during virus replication may encourage emergence and release of variants seeking the immune-privileged organs in an attempt to escape the inopportune and exasperating inopportune impact of cytotoxic T cells ${ }^{4}$. It is thus best to rely on the innate immunity defenses, notably the cytoplasmic RIG-1, which is capable of prohibiting virus replication and cell destruction, and only vaccinate people suffering impaired innate immunity, preferably with a protein vaccine based on S1, like Corbevax $\underline{3-5}$.

\section{References}

1. Ledford, H. Nature News doi: https://doi.org/10.1038/d41586-022-00063-0 (2022).

2. Klein, J., Sato, A. N. Engl. J Med. doi: 10.1056/NEJM200009073431006 (2000).

3. Tallima, H., El Ridi, R. Preprint at Authorea https://doi.10.22541/au.162731253.34527848/v1(2021).

4. Tallima, H., El Ridi, R. Preprint at Open Science Forum http://doi.org/10.31219/osf.io/v5b4k (2021).

5. Corbevax. Wikipidea. https://en.wikipedia.org/wiki/Corbevax

Hatem Tallima, Ph.D., The American University in Cairo, New Cairo 11835, Cairo, Egypt; htallima@aucegypt.edu. ORCID ID: 0000-0003-3714-641X. Mobile: 00201006672097

Rashika El Ridi, Ph.D., D.Sc., Faculty of Science, Cairo University, 12613 Giza, Egypt; rashika@sci.cu.edu.eg. ORCID ID: 0000-0001-5094-6994. Mobile: 0020\1095050 888

The authors affirm they have no conflict of interests. 